\title{
Hemolytic uremic syndrome associated with Escherichia coli 0157:H7 infection in older adults: a case report and review of the literature
}

\author{
Heidi Ko ${ }^{1 *}$, Hossein Maymani ${ }^{2}$ and Cristhiam Rojas-Hernandez ${ }^{3}$
}

\begin{abstract}
Background: Hemolytic uremic syndrome associated with Shiga toxin-producing Escherichia coli O157:H7 has been widely known as a common cause of acute renal failure in children. There are only a few reports of sporadic Shiga toxin-producing Escherichia coli-hemolytic uremic syndrome in adults in the USA. Analyses from the 2011 outbreak of hemolytic uremic syndrome associated with Escherichia coli O104:H4 reported that mortality rates are highest in those patients with age $>60$-years old. Therefore, recognizing Shiga toxin-producing Escherichia coli-hemolytic uremic syndrome in older people can help early introduction of the appropriate therapy.

Case presentation: We describe an 86-year-old Caucasian woman, initially treated as suspected thrombotic thrombocytopenic purpura, with worsening neurological and renal functions despite plasmapheresis (plasma exchange). A subsequent normal ADAMTS13 activity level and positive stool sample for Escherichia coli O157:H7 confirmed the diagnosis of Shiga toxin-associated hemolytic uremic syndrome. We shifted our management towards aggressive supportive care. Despite conventional treatment, hemolytic uremic syndrome unfortunately led to her death.

Conclusions: Our case demonstrates the importance of recognizing Shiga toxin-producing Escherichia coli-hemolytic uremic syndrome as an etiology of microangiopathic hemolytic anemia in older people. According to the current literature, supportive care is the best approach for Shiga toxin-producing Escherichia coli-hemolytic uremic syndrome. Therapies such as plasma exchange and eculizumab (a complement inhibitor) are not shown to be effective in Shiga toxin-producing Escherichia coli-hemolytic uremic syndrome. There is a dire need to continue research to find better treatment options in this disease entity with a high mortality, particularly in older people.
\end{abstract}

Keywords: Case report, Hemolysis, Microangiopathy, Elderly

\section{Background}

Microangiopathic hemolytic anemia (MAHA) results in erythrocyte fragmentation, elevated lactate dehydrogenase (LDH) levels, and microvascular occlusion. MAHA with normal ADAMTS13 activity level consists of a spectrum of disorders that includes hemolytic uremic syndrome (HUS) [1]. HUS can be further categorized into two different subsets: classical or typical HUS associated with Shiga

\footnotetext{
* Correspondence: heidi.chwan.ko@uth.tmc.edu

${ }^{1}$ Department of Internal Medicine, The University of Texas Health Science

Center, Houston, TX, USA

Full list of author information is available at the end of the article
}

toxin-producing Escherichia coli (STEC) or invasive pneumococcal infection, and atypical HUS (aHUS) that is not related to STEC and is driven by complement activation and dysregulation.

HUS associated with E. Coli O157:H7 has been widely recognized as one of the main causes of acute renal failure in children. Supportive care and dialysis have been previously known as the mainstream therapy in STECHUS in children. However, there are not many cases of STEC-HUS reported in adults worldwide. The report of an outbreak of HUS associated with STEC O104:H4 in Germany in 2011 showed the highest rate of mortality in 
adults $>60$-years old [2]. The rarity with which this illness is encountered in adults often delays diagnosis and limits treatment options for this illness.

In this case report, we demonstrate a case of an 86-yearold woman affected by STEC-HUS which is an illness more common in children. Recognizing HUS in older people and differentiating it from other MAHA entities can help early diagnosis and treatment.

\section{Case presentation}

An 86-year-old Caucasian woman was transferred to our medical intensive care unit from another medical facility to provide a higher level of care for thrombotic microangiopathy and acute renal failure. She presented to an emergency department with an acute onset of abdominal pain and bright red blood per rectum. She reportedly had some breathing difficulty. A computed tomography (CT) of her abdomen had shown nonspecific findings suggestive of colitis and therapy had been started with levofloxacin and metronidazole. Urine studies did not have features of urinary tract infection. She was intubated electively at the other hospital due to altered mental status and need for supplemental oxygen prior to her transfer. Also of note, her family reported that she had some difficulties with her speech; this was in the form of difficulties with word-finding alongside slurring of speech. She had no other neurological deficits at that time. A diagnosis of MAHA with a suspicion for thrombotic thrombocytopenic purpura (TTP) was made and she was transferred to our hospital for a higher level of medical care. On her arrival at our institution, she continued to receive metronidazole, cefepime, and tigecycline.

She had medical comorbidities of essential hypertension, hyperlipidemia, and hypothyroidism. She had no reported history of infectious, autoimmune, or hematologic diseases. On physical examination, remarkable skin findings of purpura and ecchymoses were noted. She was intubated and coarse breath sounds were appreciated on examination.

The laboratory data revealed an elevated creatinine, markedly elevated serum LDH, and markedly reduced haptoglobin levels. Her serum ferritin was $739.8 \mathrm{ng} / \mathrm{mL}$, transferrin saturation was $42 \%$; her cobalamin and folate were within normal limits. A peripheral blood smear (Fig. 1) showed thrombocytopenia and features of MAHA (Table 1).

We approached the case as MAHA with a high suspicion for TTP. However, STEC-HUS was also high in our differential diagnosis since she initially had bloody diarrhea. We decided to start plasma exchange (PLEX) alongside methylprednisolone $1 \mathrm{mg} / \mathrm{kg}$ twice daily while awaiting ADAMTS13 activity and Shiga-toxin assays. She also underwent hemodialysis for acute renal failure. After an initial improvement in hemolysis and thrombocytopenia, her laboratory and clinical parameters of MAHA failed to improve in subsequent days of hospitalization. Three days later, ADAMTS13

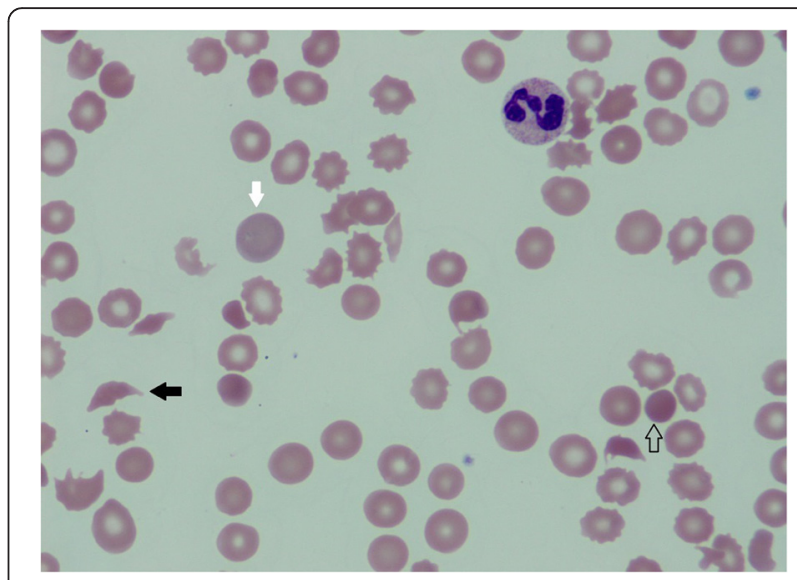

Fig. 1 Peripheral blood smear exhibiting features of microangiopathy and marked thrombocytopenia. Severe anemia with schistocytes (black solid arrow), reticulocytosis (white arrow), and spherocytes (empty arrow)

activity level was reported as $92 \%$ (normal range, 68 to $163 \%$ activity); her stool sample was positive for STEC and a culture was positive for E. coli O157:H7. These findings were consistent with a diagnosis of STEC-HUS; therefore we recommended discontinuation of corticosteroid and plasmapheresis. We had a lengthy discussion with the family members regarding the limited efficacy of available treatment options. Given her worsening clinical condition, the family decided on palliative measures; she died on her fourth day of hospitalization at our institution.

\section{Discussion}

STEC-HUS is a life-threatening illness that is characterized by MAHA, thrombocytopenia, and acute renal injury which affects 6 to $9 \%$ of STEC infections [3]. Less commonly, neurological involvement is also found to be associated with STEC-HUS infection. The pathophysiology behind STEC causing organ damage is through vasculoendothelial injury. Shiga toxin (Stx) is released by these toxic strains of bacteria into the gastrointestinal tract system and gets absorbed into the systemic circulation where it binds to globotriaosylceramide (Gb3) on the surface of vascular endothelial cells in different target organs, particularly the kidney and brain. Stx injures endothelial cells by inhibiting protein synthesis, inducing broad inflammatory response, and releasing cytokines and chemokines. This endothelial cell injury increases risk of thrombosis and organ damage [4]. Shiga toxin also activates the alternative pathway of complement system by binding to factor $\mathrm{H}$ proteins, known as complement control proteins, and reducing their cell surface activity [5]. Furthermore, Stahl et al. studied the effects of STEC on complement system and illustrated the increased binding of microparticles from platelets and 
Table 1 Changes in laboratory values during the clinical course

\begin{tabular}{|c|c|c|c|c|c|c|c|c|c|c|c|c|}
\hline Time & $\begin{array}{l}\text { WBC } \\
\left(\times 10^{3} / \mathrm{uL}\right)\end{array}$ & $\begin{array}{l}\text { Hemoglobin } \\
\left(\times 10^{3} / \mathrm{uL}\right)\end{array}$ & $\begin{array}{l}\text { Platelet } \\
\text { count } \\
\left(\times 10^{3} / \mathrm{UL}\right)\end{array}$ & $\begin{array}{l}\text { Haptoglobin } \\
\text { (mg/dL) }\end{array}$ & $\begin{array}{l}\mathrm{LDH} \\
(\mathrm{u} / \mathrm{L})\end{array}$ & $\begin{array}{l}\text { Reticulocyte } \\
\text { count }\end{array}$ & $\begin{array}{l}\text { T. Bilirubin } \\
\text { (mg/dL) }\end{array}$ & $\begin{array}{l}\text { Creatinine } \\
(\mathrm{mg} / \mathrm{dL})\end{array}$ & $\begin{array}{l}\text { BUN } \\
\text { (mg/dL) }\end{array}$ & INR & PTT & Fibrinogen \\
\hline $\begin{array}{l}\text { Initial presentation } \\
\text { at another hospital }\end{array}$ & 11 & 11.1 & 30 & $<3.0$ & 2391 & $3.70 \%$ & 2.2 & 2.49 & 73 & 1.20 & 26.0 & 577 \\
\hline $\begin{array}{l}\mathrm{HO} \text {, ICU admission } \\
\text { at our center }\end{array}$ & 7.6 & 6.4 & 57 & NA & 4576 & $6.20 \%$ & 1 & 6.8 & 112 & 1.43 & 35.8 & 412 \\
\hline $\begin{array}{l}\mathrm{H} 1,1 \text { day after } \\
\text { receiving } \\
\text { plasmapheresis }\end{array}$ & 7.4 & 7.9 & 62 & NA & 992 & NA & 1 & 5.79 & 104 & 1.36 & 30.9 & NA \\
\hline $\begin{array}{l}\mathrm{H} 2,2 \text { days after } \\
\text { receiving } \\
\text { plasmapheresis }\end{array}$ & 14.9 & 8.2 & 58 & NA & 1639 & $4.90 \%$ & 1.5 & 6.22 & 122 & NA & NA & NA \\
\hline $\begin{array}{l}\mathrm{H} 3 \text {, day stopped } \\
\text { plasmapheresis }\end{array}$ & 16.8 & 8.9 & 33 & NA & 2489 & $4.10 \%$ & 2.1 & 4.78 & 117 & 1.40 & 38.8 & 148 \\
\hline
\end{tabular}

Timeline of patient's clinical course and hemolysis laboratory data changes with interventions. $\mathrm{H} 0$ : hospital day 0 ; $\mathrm{H} 1$ : hospital day 1 ; $\mathrm{H} 2$ : hospital day 2; $\mathrm{H} 3$ : hospital day 3, NA: not available. BUN blood urea nitrogen, ICU intensive care unit, INR international normalized ratio, $L D H$ lactate dehydrogenase, $P T T$ partial thromboplastin time, $T$. total, WBC white blood cell

monocytes in the plasma of patients with HUS to C3 and $\mathrm{C} 9$ complement factors, thereby activating them, during the acute phase of the illness causing inflammatory and prothrombotic changes in different organs [6].

The usual presentation of STEC-HUS starts with a prodromal illness with abdominal pain, vomiting, and diarrhea that precedes MAHA, thrombocytopenia, and acute kidney injury (AKI) by 5 to 10 days. MAHA is characterized by hemoglobin levels usually less than $8 \mathrm{~g} / \mathrm{dL}$, negative Coombs test, elevated serum indirect bilirubin concentration, elevated serum LDH level, decreased serum haptoglobin concentration and a peripheral blood smear showing a large number of schistocytes and reticulocytes and nucleated red blood cells. Thrombocytopenia is characterized by a platelet count below $150,000 / \mathrm{mm}^{3}$. AKI is much harder to define since the severity of renal involvement in STECHUS varies from hematuria and proteinuria to development of severe AKI and oliguria requiring dialysis. Studies have shown that about $50 \%$ of patients with HUS require dialysis during the acute phase $[5,7]$. There can be additional organ involvements in HUS, with 20 to $25 \%$ of patients with HUS developing life-threatening neurological involvement with severe neurological symptoms such as lethargy, apnea, coma, seizures, cortical blindness, and hemiparesis [3]. Brain magnetic resonance imaging in patients with severe neurological involvement revealed abnormal findings in the basal ganglia, thalami, and brainstem [8]. Severe central nervous system (CNS) involvement has been shown to be associated with higher mortality [4].

Although we are more familiar with the occurrence of the disease in children, there have now been a few studies recognizing the occurrence of STEC-HUS in adults. Gould et al. analyzed data from Foodborne Diseases Active Surveillance Network (FoodNet) populationbased surveillance for E. Coli O157:H7 infection and for HUS from the years 2000 to 2006 in eight different states in the USA to further explore demographic risk factors for the development of STEC-HUS and to evaluate mortality rates associated with STEC-HUS by age. From their data analyses, they reported that young females of $<5$-years old had the highest risk for developing HUS following $E$. Coli O157:H7 infection, and people $>60$-years old had the highest mortality rate associated with E. Coli O157:H7 infection with or without HUS [9]. In the 2011 outbreak of HUS associated with E. Coli O104:H4 in Germany, the frequency of neurological symptoms was higher in adults than in children which led to higher mortality rates. It was found that deaths occurred acutely during initial hospitalization mainly due to neurological damage with symptoms consistent with cerebral edema and infarction, sepsis, and electrolyte disturbances [2].

In addition to basic supportive care, multiple treatment modalities ranging from antithrombotic agents, PLEX, tissue-type plasminogen activator, oral Stxbinding agent, and eculizumab have been studied in the management of STEC-HUS. We will mainly focus on PLEX and eculizumab therapy in this discussion as they are the two most frequently studied therapies in HUS. PLEX has been attempted as treatment for STEC-HUS. The rationale behind PLEX use in STEC-HUS would be to remove Shiga-like toxin and prothrombotic factors caused by the toxin and inflammatory mediators, and replace them with coagulation, tissue and complement factors [6]. According to the 2010 guidelines of the American Society for Apheresis, PLEX is listed as one of the recommended treatments for STEC-HUS [10]. However, analysis of the 2011 E. Coli O104:H4-associated HUS outbreak in Germany proposed that general supportive care has the same clinical outcome as PLEX in patients of all ages [2]. In our case presentation, we learned that PLEX led to an initial improvement in hemolysis and consumptive thrombocytopenia. PLEX 
should be initiated early when MAHA is suspected as it is often difficult to differentiate HUS from TTP and there is a time lag to obtain the results for ADAMTS13 activity assay and stool studies [1].

Another therapy that has been studied in STEC-HUS in children is eculizumab, a monoclonal antibody to C5 complement factor blocking complement activation. Eculizumab has been shown to be an effective drug in treatment of complement-mediated HUS (aHUS). There have been case series which have demonstrated the benefit of using eculizumab in children with STEC-HUS and CNS involvement $[11,12]$. On the contrary, other studies reported that there was no benefit seen with use of eculizumab in addition to standard medical care in patients (both adults and children) affected in the 2011 outbreak of E. Coli O104:H4 [2, 4]. There is, however, a lack of data supporting the use of eculizumab in the older population with STEC-HUS. Further randomized controlled trials are needed to identify whether, when, and in which STEC-HUS cases eculizumab administration is beneficial. The most effective treatment in terms of cost and safety was found to be general supportive care with volume resuscitation, renal replacement therapy, parenteral nutrition, and transfusion of blood products. Despite these therapeutic measures, STEC-HUS is associated with a high risk of mortality especially in older patients $[2,13]$.

\section{Conclusions}

This case demonstrates the importance of recognizing STEC-HUS as an etiology of MAHA in older people. Currently, literature data support the view that supportive care is the best approach for STEC-HUS. Conventional therapies for other MAHA, such as PLEX and eculizumab, are not shown to be effective in STEC-HUS. There is a dire need to continue research and randomized controlled trials to find better treatment options for STEC-HUS.

\section{Acknowledgements}

We would like to thank Dr Vahid Afshar-Kharghan for his assistance in revising the draft report to completion.

\section{Authors' contributions}

CRH was the lead physician responsible for the clinical care of the case, supplied all clinical details and helped revise the draft report to completion. HK jointly conceived the idea for the article (with HM), searched the background literature, wrote the report and refined the text in accordance with comments from $\mathrm{CRH}$ and $\mathrm{HM}$. HM received initial notification of the case and advised on clinical interpretation of the presenting details and helped revise the draft. All authors read and approved the final manuscript.

\section{Competing interests}

The authors declare that they have no competing interests.

\section{Consent for publication}

The patient in the case report is deceased and written informed consent was obtained from the patient's next-of-kin for publication of this case report and accompanying images. A copy of the written informed consent is available for review by the Editor-in-Chief of this journal.

\section{Author details}

Department of Internal Medicine, The University of Texas Health Science Center, Houston, TX, USA. ${ }^{2}$ Division of Internal Medicine, Section of Medical Oncology and Hematology, The University of Texas MD Anderson Cancer Center, Houston, TX, USA. ${ }^{3}$ Division of Internal Medicine, Section of Benign Hematology, The University of Texas MD Anderson Cancer Center, Houston, TX, USA.

Received: 28 March 2016 Accepted: 2 June 2016

Published online: 15 June 2016

\section{References}

1. Brodsky RA. Complement in hemolytic anemia. Hematology Am Soc Hematol Educ Program. 2015;2015(1):385-91. doi:10.1182/asheducation-2015.1.385.

2. Kielstein JT, Beutel G, Fleig S, Steinhoff J, Meyer TN, Hafer C, et al. Best supportive care and therapeutic plasma exchange with or without eculizumab in Shiga-toxin-producing E. coli 0104:H4 induced haemolytic-uraemic syndrome: an analysis of the German STEC-HUS registry. Nephrol Dial Transplant. 2012;27(10):3807-15. doi:10.1093/ndt/gfs394.

3. Siegler RL. Spectrum of extrarenal involvement in postdiarrheal hemolytic-uremic syndrome. J Pediatr. 1994;125(4):511-8.

4. Trachtman H, Austin C, Lewinski M, Stahl RA. Renal and neurological involvement in typical Shiga toxin-associated HUS. Nat Rev Nephrol. 2012:8(11):658-69. doi:10.1038/nrneph.2012.196.

5. Thurman JM, Marians R, Emlen W, Wood S, Smith C, Akana H, et al. Alternative pathway of complement in children with diarrhea-associated hemolytic uremic syndrome. Clin J Am Soc Nephrol. 2009;4(12):1920-4. doi:10.2215/CJN.02730409.

6. Stahl AL, Sartz L, Karpman D. Complement activation on platelet-leukocyte complexes and microparticles in enterohemorrhagic Escherichia coli-induced hemolytic uremic syndrome. Blood. 2011;117(20):5503-13. doi:10.1182/blood-2010-09-309161.

7. Gerber A, Karch H, Allerberger F, Verweyen HM, Zimmerhackl LB. Clinical course and the role of shiga toxin-producing Escherichia coli infection in the hemolyticuremic syndrome in pediatric patients, 1997-2000, in Germany and Austria: a prospective study. J Infect Dis. 2002;186(4):493-500. doi:10.1086/341940.

8. Bauer A, Loos S, Wehrmann C, Horstmann D, Donnerstag F, Lemke J, et al. Neurological involvement in children with E. coli 0104:H4-induced hemolytic uremic syndrome. Pediatr Nephrol. 2014;29(9):1607-15. do: 10.1007/s00467-014-2803-x.

9. Gould LH, Demma L, Jones TF, Hurd S, Vugia DJ, Smith K, et al. Hemolytic uremic syndrome and death in persons with Escherichia coli 0157:H7 infection, foodborne diseases active surveillance network sites, 2000-2006. Clin Infect Dis. 2009:49(10):1480-5. doi:10.1086/644621.

10. Szczepiorkowski ZM, Winters JL, Bandarenko N, Kim HC, Linenberger ML, Marques MB, et al. Guidelines on the use of therapeutic apheresis in clinical practice - evidence-based approach from the Apheresis Applications Committee of the American Society for Apheresis. J Clin Apher. 2010;25(3):83-177. doi:10.1002/jca.20240.

11. Colic E, Dieperink H, Titlestad K, Tepel M. Management of an acute outbreak of diarrhoea-associated haemolytic uraemic syndrome with early plasma exchange in adults from southern Denmark: an observational study. Lancet. 2011;378(9796):1089-93. doi:10.1016/S0140-6736(11)61145-8.

12. Rosales A, Hofer J, Zimmerhackl LB, Jungraithmayr TC, Riedl $M$, Giner $T$, et al. Need for long-term follow-up in enterohemorrhagic Escherichia coliassociated hemolytic uremic syndrome due to late-emerging sequelae. Clin Infect Dis. 2012;54(10):1413-21. doi:10.1093/cid/cis196.

13. Hickey CA, Beattie TJ, Cowieson J, Miyashita Y, Strife CF, Frem JC, et al. Early volume expansion during diarrhea and relative nephroprotection during subsequent hemolytic uremic syndrome. Arch Pediatr Adolesc Med. 2011;165(10):884-9. doi:10.1001/archpediatrics.2011.152. 